\title{
Coexistence of osteopoikilosis with seronegative spondyloarthritis and Raynaud's phenomenon: first case report with evaluation of the nailfold capillary bed and literature review
}

\author{
B. Ruaro, A. Sulli, E. Alessandri, F. Ravera, M. Cutolo \\ Research Laboratory and Academic Unit of Clinical Rheumatology, \\ Department of Internal Medicine, University of Genoa, Italy
}

\begin{abstract}
Osteopoikilosis (OPK) is a rare autosomal dominant bone disorder characterized by numerous hyperostotic areas that tend to localize in periarticular osseous regions. It is usually asymptomatic and is often diagnosed incidentally during X-rays. OPK may be an isolated finding or associated with other pathologies, e.g. skin manifestations, rheumatic and/or skeletal disorders. We report a literature review and, for the first time, the coexistence of OPK with seronegative spondyloarthritis and Raynaud's phenomenon in a 48-year old female. To the best of our knowledge, this is the first case of OPK studied by videocapillaroscopy, demonstrating the absence of specific microvascular abnormalities of nailfold capillaries.
\end{abstract}

SUMMARY

Key words: Osteopoikilosis, spondyloarthritis, Raynaud's phenomenon, nailfold videocapillaroscopy.

Reumatismo, 2012; 64 (5): 335-339

\section{INTRODUCTION}

steopoikilosis (OPK also known as disseminated condensing osteopathy, spotted bones, osteopecilia) is a rare hereditary bone disease (1). It is characterized by the presence of hyperostotic spots, preferentially localized in the epiphyses and/or metaphyses of the long bones, in the carpal and tarsal bones (2). The first case was reported in 1915 by Albers-Schönberg (3-5). There are three clinical variants of OPK. The most common is the speckled type, followed by the striated form, while the mixed form is rarer. OPK has an estimated prevalence of 1 per 50,000 while the incidence in skeletal X-rays is reported to be 1 per 20,000 $(1,6)$. Nowadays, OPK is known to be an autosomal dominant hereditary disease caused by heterozygous mutations in the LEMD3 gene (locus 12q14). LEMD3 encodes an inner nuclear membrane protein that seems to play a role in bone morphogenetic protein signaling
$(7,8)$. Apparently there is also a sporadic form (1, 7-9).

There is no difference between gender, with males and females being equally affected; however, other Authors report that this pathology is more predominant in the male population (ratio 2:1) $(1,10)$. OPK is often observed incidentally when X-rays are taken for trauma. Although this pathology is usually asymptomatic, $15-20 \%$ of patients may have slight articular pain and/ or joint effusion (10-12). Specific diagnosis is based exclusively on X-ray as usually no haematochemical alterations are involved. Bone biopsy reveals focal condensations of compact lamellar bone within the spongiosa (13).

We report a literature review and, for the first time, the coexistence of OPK with seronegative spondyloarthritis and Raynaud's phenomenon in a 48-year old female. To the best of our knowledge, this is the first case of OPK studied by nailfold videocapillaroscopy (NVC). $\overline{\text { Corresponding author: }}$ Alberto Sulli

Research Laboratory and Academic Unit of Clinical Rheumatology Department of Internal Medicine University of Genoa

V.le Benedetto XV, 6 - 16132 Genova, Italy E-mail: albertosulli@unige.it 


\section{CASE REPORT}

A 48-year old female (CM) presented to our Rheumatology Outpatient Service in February 2010 complaining of diffused joint pain, above all in the lumbar and sacral region (with nocturnal exacerbation), wrists, shoulders and knees, of a 3 -year duration. Her condition had previously been controlled with analgesic drugs by her G.P.. She had also been affected by Raynaud's phenomenon for ten years and complained of morning stiffness in the hands that lasted about 30 minutes. She had a history of slipped disk in 2004 and a diagnosis of hip osteoarthritis in 2007 that had led to left hip prosthesis in the same year. In 2009, when she was 47 years old, she had an X-ray for pain in the pelvic girdle and a diagnosis of OPK was made on the basis of the presence of widespread round, oval, focal radiodense lesions lo-

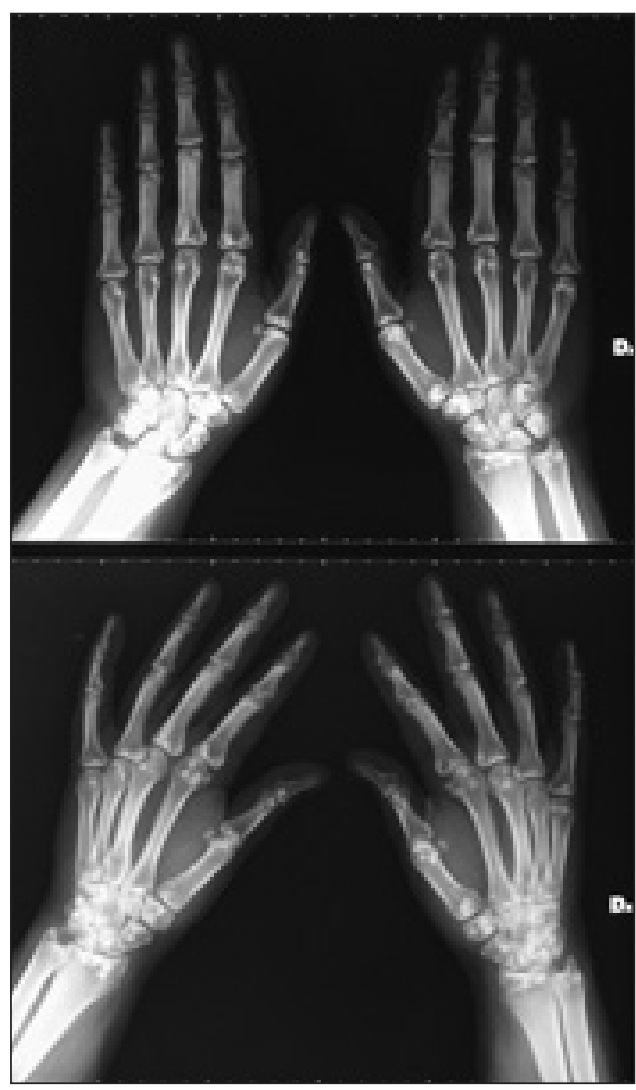

Figure 1 - X-ray of hands and wrists: the presence of speckled osteopoikilosis in the radial, ulnar and metacarpal bones is seen. calized in the pubic region, distal epiphyses and metaphyses of the left femur and proximal tibia.

She was given a thorough clinical examination, and laboratory tests were carried out. The clinical examination revealed a normal range of motion of the peripheral joints, a slight swelling of her wrists, and painfulness in the articular areas mentioned above. Her range of motion of the spine was normal, but there was a mild tenderness at the sacroiliac joints. There were no skin lesions. Raynaud's phenomenon was not associated to sclerodactily.

Hand and knee X-rays were negative for both calcium deposits and bone erosions. The X-ray of her hands and wrists confirmed the presence of speckled OPK in the radial, ulnar and metacarpal bones (Fig. 1). X-rays of the spine demonstrated interapophyseal sclerosis of the joints in the lumbar region along with slight convexed right lumbar scoliosis in hyperlordosis. A pelvic girdle X-ray documented a bilateral sclerosis of the sacroiliac joints, along with secondary osteoarthritic manifestations. There were also round focal radiodense lesions (hyperostotic spots) localized in the proximal epiphyses and metaphyses of the left hip and in the pubic region (Fig. 2). Magnetic resonance imaging confirmed the presence of arthritic manifestations at sacroiliac joints, more evident on the right side (bilateral subchondral bone marrow edema and sclerosis, bilateral irregularities of sacroiliac joints with erosions at the right iliac site). There was an increase in inflammatory marker erythrocyte sedimentation rate and $\mathrm{C}$-reactive protein ( $38 \mathrm{~mm} / \mathrm{L} \mathrm{h}$ and $23 \mathrm{mg} / \mathrm{L}$, respectively). Other laboratory tests were unremarkable, including renal and liver function tests, rheumatoid factor, anti-cyclic citrullinated peptide antibodies, antinuclear antibodies, and human leukocyte antigen B27. The osseous markers, parathormone, calcium, phosphorus, vitamin D and alkaline phosphatase were all within normal ranges. Serology for chlamydia and mycoplasma, and urine cultures for ureaplasma urealyticum and mycoplasma hominis were all negative. 


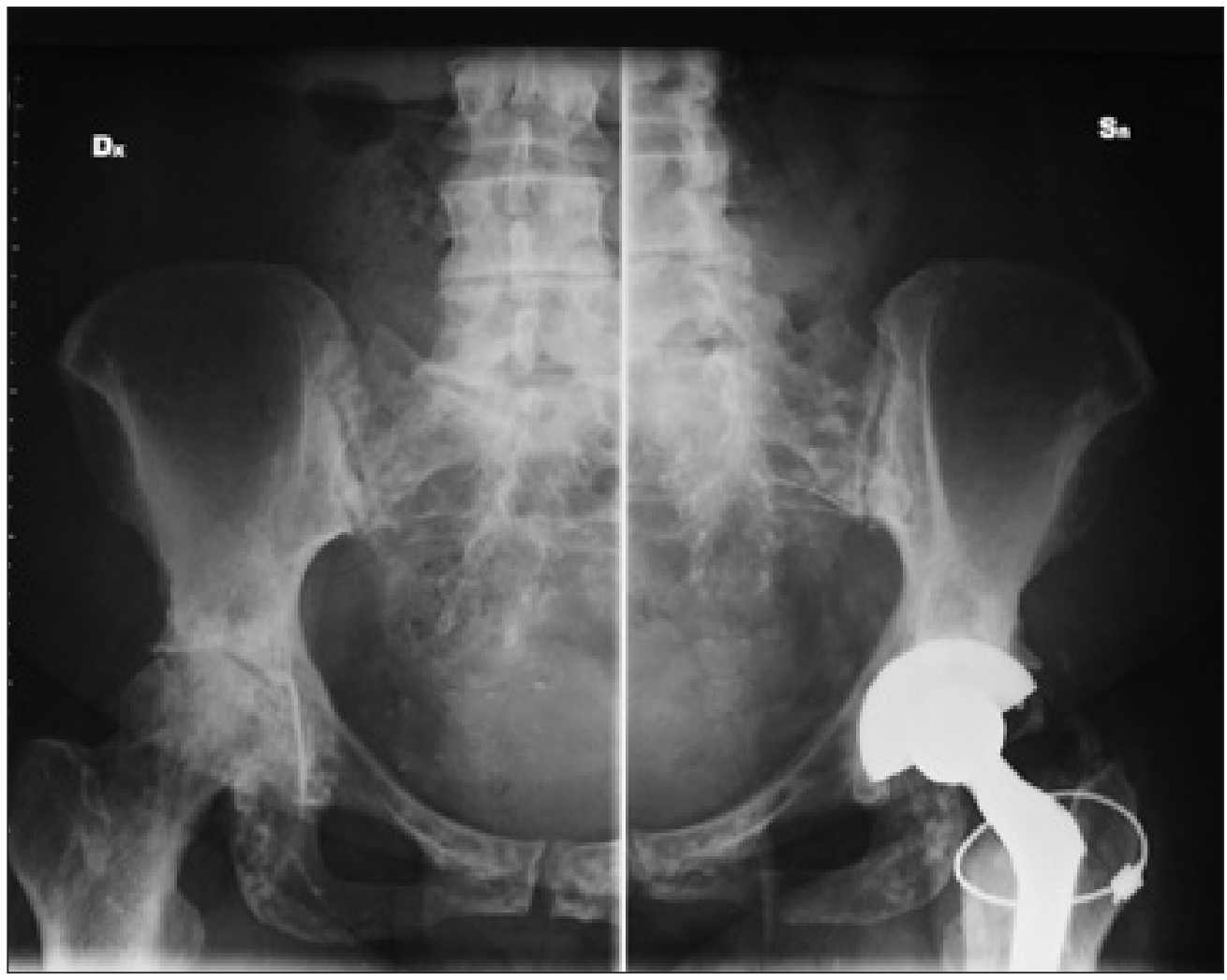

Figure 2 - X-ray of pelvis and hip: the presence of oval and round, multiple, regular and symmetrical radiodense lesions of osteopoikilosis, localized around pelvis and hip is seen.

A diagnosis of seronegative spondyloarthritis was made on the basis of ASAS criteria (14). Treatment was started with prednisone at a dosage of $5 \mathrm{mg} /$ day for two months, with limited benefit. Therefore, the dosage of prednisone was increased to $7.5 \mathrm{mg} /$ day and methotrexate was added at $10 \mathrm{mg}$ weekly. Symptoms gradually improved over a 2-month period and inflammatory markers also progressively normalized. NVC was performed to evaluate Raynaud's phenomenon (15). Results were in the normal range for capillary morphology without any scleroderma-pattern: 10

Figure 3 - (A-B) Normal nailfold videocapillaroscopy in patient CM: 10 capillaries per linear millimetre are seen with a perpendicular distribution to the periungueal edge. Capillaries are U-shaped, sometimes tortuous or with irregular ectasias; neither giant capillaries nor capillary ramifications are present.

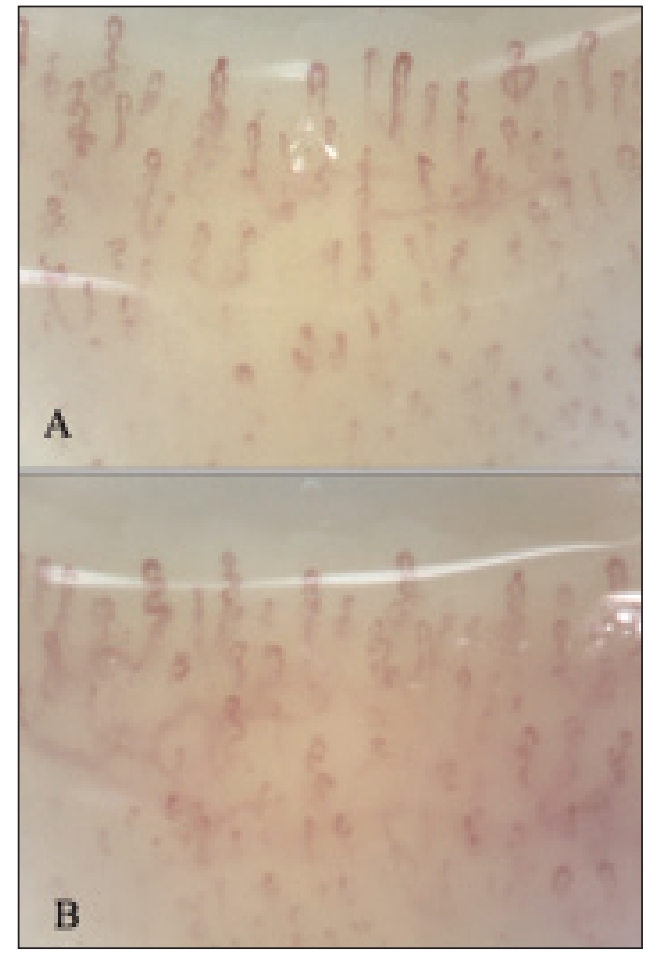


capillaries per linear millimetre were present with a perpendicular distribution to the periungueal edge, without pericapillary oedema. The only abnormalities observed were tortuous capillaries and rare irregular capillary ectasias, without giant capillaries (Fig. 3A and B). At time of writing (20 months after the first visit) NVC status was unchanged.

\section{DISCUSSION}

Osteopoikilosis is known to be an autosomal dominant hereditary disease characterized by sclerosing bone dysplasia, even if there was no documented family history for the case reported herein.

There are three clinical types of OPK: speckled, striated, and mixed. Our patient was affected by the speckled type characterized by widespread round and oval focal radiodense lesions and hyperostotic spots (Fig. 1 and 2).

The most frequently affected sites are the bones of hands (phalanges, carpal, metacarpal) and feet (phalanges, tarsal, metatarsal). Less frequently involved sites include the legs (femur), the axial skeleton (pelvis, sacrum), and arms (radius, ulna), with rare involvement of the ribs, clavicles, skull and vertebrae $(6,16,17)$. Our patient's lesions were localized in both proximal and distal epiphyses, metaphyses of the hip and in the proximal tibia, with symmetrical spotted lesions at wrists (radial, ulnar and metacarpal). In speckled OPK the lesions appear nodular, diffuse and symmetrical; parallel and longitudinal striae are observed in striated OPK while there is a combination of striae and mottling in mixed OPK (1). Our patient's lesions were multiple, oval and round, regular and symmetrical, around her wrists, pelvis and hip (Fig. 1 and 2). The diagnosis of OPK is usually incidental, made in concomitance with X-rays for other pathologies. In fact, only $15-20 \%$ of patients may have slight articular pain and/or joint effusion (10, 17-19). Differential diagnosis in cases of widespread focal round or oval radiodense lesions is between osteoblastic metastases, primary bone tumors, mastocytosis, tuberous sclerosis and synovial chondromatosis. In the presence of only a few striae, the differential diagnosis involves melorheostosis. As far as osteoblastic metastasis is concerned, these are more frequently observed in the axial skeleton and are not uniform but are asymmetric, and vary in size with osseous destruction and positive scintigraphy findings; OPK has symmetric, regular oval or rounded lesions, localized predominantly around the joints (19-25).

No therapy is required, except in particular cases in which either the patient complains of severe articular pain (for which analgesic drugs are indicated) or symptoms associated to other pathologies are presented. In our case, no pain relief was obtained from non-steroidal anti-inflammatory drug treatment until we considered the associated pathology (spondyloarthrits) and modified the treatment regimen accordingly; remission of symptoms was then achieved. Occasionally, OPK may coexist with dermatological pathologies (scleroderma, dermatofibrosis lenticularis disseminata, keloid formation, plantar and palmar keratoma), heart or renal malformations, endocrine disorders, or skeletal diseases (exostosis, spinal stenosis, chondrosarcoma, osteosarcoma) (18-21, 26). OPK has also been described in association with rheumatoid arthritis, reactive arthritis, discoid lupus erythematosus, familial Mediterranean fever, psoriatic arthritis, or fibromyalgia (10-13).

In conclusion, the present work reports for the first time the case of a patient affected by OPK associated with spondyloarthritis and Raynaud's phenomenon. NVC examination helped exclude the presence of a scleroderma-pattern of microangiopathy. Furthermore, this is the first paper that has analyzed the nailfold capillary bed in a patient affected by OPK, demonstrating the absence of specific microvascular abnormalities in this clinical condition.

We believe that, in cases in which a diagnosis of OPK is made, particular attention must be paid not only to the differential diagnosis, but also to the possible association 
of the disease with other pathologies. This will allow the best therapeutic regime to be selected, particularly in the presence of pain.

\section{Conflict of interest}

All Authors declare no conflict of interest.

\section{REFERENCES}

1. Canepa G, Moroteaux P, Pietrogrande V. Dismorphic syndrome and constitutional disease of the skeleton. Ed. Piccin. 2001; Vol. II: 1393-7.

2. Barisone D, Neumaier F. Contributo allo studio clinico-radiologico dell'osteopecilia. Minerva Ortopedica. 1956; 7: 219-26.

3. Hoeffel JC, Pichene A, Galloy MA, Mainard L. L'osteopathie striée. Ann Pediatr. 1993; 40: 285-90.

4. Ferri S, Grosso F, Roversi R, Marsigli R. Reumatologia. Casistica reumatologica non comune. Minerva Medica. 1983; 74: 219-26.

5. Horan FT, Beighton PH. Osteopathia striata with cranial sclerosis. An autosomal dominant entity. Clinical Genetics. 1978; 13: 201-6.

6. Kaparov A, Uludag M, Sari H, Akarirmak U. De Quervain's Syndrome associated with osteopoikilosis: a case report and review of the literature. Rheumatol Int. 2011; 31: 809-13.

7. Baasanjav S, Jamsheer A, Kolanczyk M, et al. Osteopoikilosis and multiple exostoses caused by novel mutations in LEMD3 and EXT1 genes resplectively - Coincidence within one family. BMC Med Genet. 2010; 11: 110.

8. Hellemans J, Preobrazhenska O, Willaert A, et al. Loss-of-function mutation in LEMD3 result in osteopoikilosis, Buschke-Ollendorff syndrome and melorheostosis. Nat Genet. 2004; 36: 1213-8.

9. Manger K, Nüsslein H, Schett G, Manger B. Rare monogenetic syndromes in rheumatology practice. Clin Rheumatol. 2009; 28: 623-30.

10. Serdaroğlu M, Capkin E, Uçüncü F, Tosun M. Case report of a patient with osteopoikilosis. Rheumatol Int. 2007; 27: 683-6.

11. Ureten K. Osteopoikilosis in a patient with rheumatoid arthritis complicated with dry eyes. Rheumatol Int. 2007; 27: 1079-82.

12. Mesci E. Coexistence of osteopoikilosis with reactive arthritis: a case report. Rheumatol Int. 2006; 26: 672-5.
13. Sari I, Simsek I, Guvenc I, et al. Osteopoikilosis coexistence with ankylosing spondylitis and familial Mediterranean fever. Rheumatol Int. 2009; 29: 321-3.

14. Rudwaleit M, van der Heijde D, Landewé R, et al. The assessment of Spondyloarthritis International Society classification criteria for peripheral spondyloarthritis and for spondyloarthritis in general. Ann Rheum Dis. 2011; 70 : 25-31.

15. Cutolo M, Sulli A, Smit V. Assessing microvascular changes in systemic sclerosis diagnosis and management. Nat Rev Rheumatol. 2010; 6: 578-87.

16. Padin-Paz EM, Diaz-Peroming JA. Osteopoikilosis in the knee. CMAJ. 2011; 183: 61.

17. Hill CH, Tan CK. Osteopoikilosis in pelvic region. CMAJ. 2011; 183:460.

18. Ozdemirel AE, Cakit BD, Erdem HR, Koc B. A rare benign disorder mimicking metastasis on radiographic examination: a case report of osteopoikilosis, Rheumatol Int. 2010; 31: 1113-6.

19. Benli IT, Akalin S, Boysan E, et al. Epidemiological, clinical and radiological aspects of osteopoikilosis. J Bone Joint Surg. 1994; 74 : 504-6.

20. Borman P, Ozoran K, Aydog` S, Cos ‘kun S. Osteopoikilosis: report of a clinical case and review of the literature. Joint Bone Spine. 2002; 69: 230-3.

21. Carpintero P, Abad JA, Serrano P, et al. Clinical features of ten cases of osteopoikilosis. Clin Rheumatol. 2004; 23: 505-8.

22. Nakamura T, Yokomizo Y, Kanda S, et al. Osteopathia striata with cranial sclerosis affecting three family members. Skeletal Radiol. 1985; 14: 267-9.

23. Cantatore FP, Carrozzo M, Loperfido MC. Mixed sclerosing bone dystrophy with features resembling osteopoikilosis and osteopathia striata. Clin Rheumatol. 1991; 10: 191-5.

24. Lagier R, Mbakop A, Bigler A. Osteopoikilosis: a radiological and pathological study. Skeletal radiol. 1984; 11: 161-8.

25. Whyte MP, Murphy WA, Fallon MD, Hahn TJ. Mixed-sclerosing-bone-dystrophy: report of a case and review of the literature. Skeletal Radiol. 1981; 6: 95-102.

26. Bicer A, Tursen U, Ozer C, et al. Coexistence of osteopoikilosis and discoid lupus erythematosus: a case report. Clin Rheumatol. 2002; 10: 405-7. 